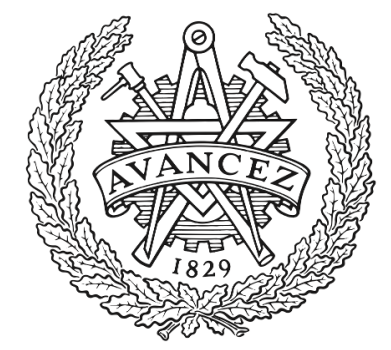

CHALMERS

UNIVERSITY OF TECHNOLOGY

\title{
Graphene plasmons: Impurities and nonlocal effects
}

Downloaded from: https://research.chalmers.se, 2023-04-26 11:30 UTC

Citation for the original published paper (version of record):

Viola, G., Wenger, T., Kinaret, J. et al (2018). Graphene plasmons: Impurities and nonlocal effects. Physical Review B - Condensed Matter and Materials Physics, 97(8).

http://dx.doi.org/10.1103/PhysRevB.97.085429

N.B. When citing this work, cite the original published paper. 


\title{
Graphene plasmons: Impurities and nonlocal effects
}

\author{
Giovanni Viola, ${ }^{1, *}$ Tobias Wenger, ${ }^{1}$ Jari Kinaret, ${ }^{2}$ and Mikael Fogelström ${ }^{1}$ \\ ${ }^{1}$ Department of Microtechnology and Nanoscience (MC2), Chalmers University of Technology, S-412 96 Göteborg, Sweden \\ ${ }^{2}$ Department of Physics, Chalmers University of Technology, S-412 96 Göteborg, Sweden
}

(Received 12 November 2017; revised manuscript received 6 February 2018; published 20 February 2018)

\begin{abstract}
This work analyzes how impurities and vacancies on the surface of a graphene sample affect its optical conductivity and plasmon excitations. The disorder is analyzed in the self-consistent Green's function formulation and nonlocal effects are fully taken into account. It is shown that impurities modify the linear spectrum and give rise to an impurity band whose position and width depend on the two parameters of our model, the density and the strength of impurities. The presence of the impurity band strongly influences the electromagnetic response and the plasmon losses. Furthermore, we discuss how the impurity-band position can be obtained experimentally from the plasmon dispersion relation and discuss this in the context of sensing.
\end{abstract}

DOI: 10.1103/PhysRevB.97.085429

\section{INTRODUCTION}

Plasmons are electromagnetic fields resonantly enhanced by oscillations in the charge density. Due the properties of graphene, in graphene plasmons exhibit low losses [1], tunable optical properties [2], strong optical field confinement [3,4], and environmental sensitivity [5-7]. This makes graphene an attractive material for next generation technologies [8] in sensing [5,9], photonics, electronics [10,11], and communication [12]. To improve device design and performance, it is crucial to extend the microscopic theory of plasmons to include nonlocal effects [13-15] together with the impact of defects and impurities [16] in the sample as well as chemical compounds deposited on the surface [17,18]. Defects and impurities may be due to the fabrication procedure, while chemical compounds can be deposited in a controlled fashion on the surface to functionalize the graphene substrate $[8,16-$ 19]. Defects and impurities are inevitably sources of losses that must be understood in order to make high-performance samples and devices, mainly by circumventing their lossproducing effects.

The behavior of plasmons in pristine graphene is by now rather well studied [20-22]. The local transport properties are investigated in a series of articles, e.g., [23-30], including effects due to the impurities, phonons, and localized charges. Phonon- and electron-electron interaction has been studied in Refs. [31,32]. The nonlocal effects in the presence of impurities or adatoms have been considered, among others, in Refs. [33-36].

First-principles studies have determined how crystal defects or atoms on the graphene surface influence the material properties [36-42]. Defects are seen to give rise to new bands whose properties depend on the density and type of defects or adsorbates. This opens the possibility to engineer the band structure of the material.

\footnotetext{
*giova.viola@gmail.com
}

While first-principles studies consider relatively small graphene supercells (on the order of $10^{2}$ atoms), many-body techniques are more suitable to describe properties in $\mu \mathrm{m}$ size devices. In this work we include impurities in a self-consistent t-matrix treatment of a uniform distribution of isotropic $(s$-wave) scattering impurities, and explore how their presence modify the optical conductivity and plasmonic behavior of graphene. In the microscopic model used here, as described in Sec. II, the nonmagnetic impurities are described as onsite, spin-preserving potentials and treated self-consistently $[24,26,43,44]$. The nonlocal transport and optical properties are investigated in Sec. III. The optical response resembles the one obtained in the relaxation-time approximation $[3,45]$ in the case of dislocations in graphene, while novel features are observed if the impurity band is detuned from the Dirac point. In particular, it is observed that an impurity band far from the Dirac point enhances the plasmon losses. Finally, we discuss how the optical response and plasmonic behavior can characterize the impurity itself (Sec. IV), within our treatment. Our work emphasizes the potential of plasmon-based sensors and of contactless characterization of samples [46]. In the following the densities of electrons and impurities are given in units of $10^{12} / \mathrm{cm}^{2}$, the energies are measured in $\mathrm{eV}, 1 \mathrm{eV}=$ $241.8 \mathrm{THz}=1239 \mathrm{~nm}$ and the conductance in units of $\sigma_{0}=$ $e^{2} /(4 \hbar)=6.085 \times 10^{-5} \mathrm{~S}=(16.4 \mathrm{k} \Omega)^{-1}$.

\section{MODEL}

Longitudinal plasmons confined at a conducting interface between two dielectrics, with relative dielectric constants $\varepsilon_{1}$ and $\varepsilon_{2}$, satisfy the dispersion relation $[3,47]$

$$
\frac{\left(\varepsilon_{1}+\varepsilon_{2}\right)}{q}+\frac{i \sigma(q, \omega)}{\varepsilon_{0} \omega}=0
$$

with the wave vector, $\boldsymbol{q}(q=|\boldsymbol{q}|)$, in the graphene plane and the angular frequency $\omega$ of the electromagnetic field. Here we assume the nonretarded limit, $q \gg \sqrt{\varepsilon_{1,2}} \omega / c$, as the light and the plasmons have a large momentum mismatch. An efficient coupling of light to plasmon modes is possible by introducing 
a dielectric grating or coupling via evanescent light modes [47] to overcome the mismatch. The nonlocal longitudinal conductivity, $\sigma(\boldsymbol{q}, \omega)=\sigma_{1}(\boldsymbol{q}, \omega)+i \sigma_{2}(\boldsymbol{q}, \omega)$, together with the dielectric environment, encodes the plasmon properties. As conductors in general are lossy, $\sigma_{1}(q, \omega)>0$, we can read from Eq. (1) that either $q$ or $\omega$ is required to be complex [32] to account for these losses. Connecting to scattering experiments, e.g., in Refs. [4,48,49], $\omega$ is the frequency of the incoming light and thus real valued which leaves the wave number $q=q_{1}+i q_{2}$ being a complex-valued quantity describing the in-plane momentum $q_{1}$ and the damping $q_{2}$ of the plasmons. For a lossless dielectric, Eq. (1) reduces to the two real equations

$$
q_{1}=\frac{\omega \varepsilon_{0}\left(\varepsilon_{1}+\varepsilon_{2}\right)}{\sigma_{2}\left(q_{1}, \omega\right)}, \quad \frac{q_{2}}{q_{1}}=\frac{\sigma_{1}\left(q_{1}, \omega\right)}{\frac{\partial}{\partial_{q_{1}}}\left(q_{1} \sigma_{2}\left(q_{1}, \omega\right)\right)},
$$

to first order in $q_{2} / q_{1}$. The ratio $q_{2} / q_{1}$ quantifies the plasmon losses and is called the plasmon damping ratio [32].

The nonlocal longitudinal conductivity $\sigma(\boldsymbol{q}, \omega)$ is evaluated from the current-current response to an external longitudinal electromagnetic field within RPA as

$$
\begin{aligned}
\chi_{j_{\mathrm{x}} j_{\mathrm{x}}}(\boldsymbol{q}, \omega)= & g_{s} g_{v} \frac{i e^{2} v_{F}^{2}}{2} \int \frac{d \boldsymbol{p} d \epsilon}{(2 \pi)} \\
& \times \operatorname{Tr}\left[\sigma_{\mathrm{x}} G^{R}(\boldsymbol{p}, \epsilon) \sigma_{\mathrm{x}} G^{K}(\boldsymbol{p}-\boldsymbol{q}, \epsilon-\omega)\right. \\
& \left.+\sigma_{\mathrm{x}} G^{K}(\boldsymbol{p}, \epsilon) \sigma_{\mathrm{x}} G^{A}(\boldsymbol{p}-\boldsymbol{q}, \epsilon-\omega)\right] .
\end{aligned}
$$

( $\operatorname{Tr}$ is the trace on the sublattice index) and the conductivity is then given as

$$
\sigma(\boldsymbol{q}, \omega)=\frac{i}{\omega} \chi_{j_{\mathrm{x}} j_{\mathrm{x}}}(\boldsymbol{q}, \omega)
$$

The conductivity [Eq. (4)] we derive from the leading order correction to the Keldysh Green's function $\delta \check{G}$ in the applied electromagnetic field (details may be found in Refs. [35,50])

$$
\delta \check{G}=\check{G} \circ[\delta \check{H}+\delta \check{\Sigma}] \circ \check{G}, \quad \check{X}=\left(\begin{array}{cc}
X^{R} & X^{K} \\
0 & X^{A}
\end{array}\right) .
$$

The microscopic details of the material are encoded in the unperturbed Green's functions $G^{R}, G^{A}$, and $G^{K}$ and the selfenergies $\Sigma^{R, A, K}$. In equilibrium we have [51] $G^{A}=\left(G^{R}\right)^{\dagger}$ and $G^{K}=f(\epsilon)\left(G^{R}-G^{A}\right)$ with $f(\epsilon)$ the Fermi distribution, where the arguments of the Green's functions, $\boldsymbol{p}$ and $\epsilon$, are occasionally omitted. Here we consider a dilute ensemble of $s$-wave scatters, smooth on the atomic scale, included via a self-consistent t-matrix method $[23,24,26,43,44]$. In this case vertex corrections which in our calculations are accounted for by the self-energy correction $\delta \check{\Sigma}$ vanish [23,52] after averaging over the isotropic impurity distribution.

Our model contains two parameters, with $V_{\text {imp }}$ being the strength, and $n_{\text {imp }}$ the density of impurities, respectively. The density $n_{\text {imp }}=N_{\text {imp }} / N$ is the number of impurities $N_{\text {imp }}$ divided by the number of unit cells $N$ in the crystal. We will use the Dirac approximation and in this scheme the energy scale is set by a cutoff $\epsilon_{c}$ related to the bandwidth, we set $\epsilon_{c}=8.2 \mathrm{eV}$ [24].
The band structure is obtained from the poles of the Green's function

$$
G^{R}(\boldsymbol{p}, \epsilon)=\sum_{\lambda= \pm} \frac{1 / 2}{\epsilon^{R}-E_{\lambda, p}-\Sigma_{\text {imp }}^{R}(\epsilon)}\left(\begin{array}{cc}
1 & \lambda \mathrm{e}^{-i \phi_{p}} \\
\lambda \mathrm{e}^{i \phi_{p}} & 1
\end{array}\right)
$$

with the self-energy

$$
\Sigma_{\mathrm{imp}}^{R}(\epsilon)=\frac{n_{\mathrm{imp}} V_{\mathrm{imp}}}{1-V_{\mathrm{imp}} \frac{1}{N} \sum_{\boldsymbol{p}} G^{R}(\boldsymbol{p}, \epsilon)} .
$$

Here $E_{\lambda, p}=\lambda v_{F} p$ is the single particle energy for the pristine graphene, $\lambda= \pm$, the band index, $v_{F}$ the electron momenta, $|\boldsymbol{p}|=p$, and $\phi_{p}=\arg \left(p_{x}+i p_{y}\right) . v_{F}$ is the Fermi velocity of graphene. The energies are measured from the Dirac point of pristine graphene. This single Dirac-cone approximation captures the physics in the regime of interest, and the degeneracy number $g_{v} g_{s}=4$ will be included at the end to include spin and valley degeneracy (intervalley and spin-flip processes are omitted).

Equation (7) is derived as an average over a distribution of identical impurities as $\Sigma_{\text {imp }}^{R}(\epsilon)=n_{\text {imp }} T_{\text {imp }}(\epsilon)$. The scattering off an impurity is described by the single impurity $T$ matrix $T_{\mathrm{imp}}$. In the Dirac-cone approximation, where the momentum sum $\frac{1}{N} \sum_{\boldsymbol{p}} G(\boldsymbol{p}, \epsilon)$ can be evaluated analytically [44], we have

$$
\Sigma_{\mathrm{imp}}^{R}(\epsilon)=\frac{n_{\mathrm{imp}}}{\frac{1}{V_{\mathrm{imp}}}-\frac{z}{\epsilon_{c}^{2}} \ln \left[\frac{-z^{2}}{\epsilon_{c}^{2}-z^{2}}\right]}
$$

with $z=\epsilon-\Sigma_{\text {imp }}^{R}(\epsilon)$, and $\epsilon_{c}$ is a cutoff that we set to the bandwidth of the graphene band structure. The poles of $\Sigma_{\mathrm{imp}}^{R}$ describe the well-known impurity states which in the limit $V_{\text {imp }} \gg \epsilon_{c}$ and $n_{\text {imp }}$ is small (i.e., $\Sigma_{\text {imp }}^{R} \rightarrow 0$ so that $z \rightarrow$ $\left.\epsilon+i 0^{+}\right)$are localized states at energies

$$
\epsilon_{\mathrm{imp}}=\frac{\epsilon_{c}^{2}}{2 V_{\mathrm{imp}}} \ln \left|\frac{\epsilon_{c}}{2 V_{\mathrm{imp}}}\right| .
$$

These low-energy impurity states are a generic feature of impurity scattering in Dirac materials [53]. At finite impurity density $n_{\text {imp }}$, the impurity state develops into a narrow metallic band around the energy $\epsilon_{\text {imp }}$ with a width,

$$
\gamma_{\mathrm{imp}} \approx \sqrt{\frac{n_{\mathrm{imp}}}{2 \ln \left|\frac{2 V_{\mathrm{imp}}}{\epsilon_{c}}\right|}} \epsilon_{c} .
$$

$\Sigma_{\text {imp }}^{R}(\epsilon)$ has a simple pole structure with a complex pole $\tilde{\epsilon}_{\text {imp }}=$ $\epsilon_{\mathrm{imp}}+i \gamma_{\mathrm{imp}}$ indicating that a distribution of impurities induces scattering resonances rather than proper long-lived states.

Self-consistent solution of equations (6) and (7) is straightforward by simple iteration. The band structure, given by $E_{\lambda, p}=\epsilon-\operatorname{Re}\left[\Sigma_{\text {imp }}^{R}(\epsilon)\right]$, is modified already for quite dilute impurity densities. The basic results are shown in Fig. 1. The impurity state $\epsilon_{\text {imp }}$ is modified into a resonance that we define as $\operatorname{Re} \Sigma_{\text {imp }}^{R}\left(\epsilon_{\text {res }}\right)=0$. The resonance $\epsilon_{\text {res }}$ is shifted towards smaller energies (absolute magnitude) compared to $\epsilon_{\text {imp }}$. There is also an impurity dependent shift of the the Dirac point $E_{\lambda, p}=0=\epsilon_{\mathrm{D}}-\operatorname{Re} \Sigma_{\text {imp }}^{R}\left(\epsilon_{\mathrm{D}}\right)$. In Fig. 2 we show how $\epsilon_{\text {res }}$ and $\epsilon_{\mathrm{D}}$ depend on the inverse of the scattering strength. The new Dirac point $\epsilon_{\mathrm{D}}$ is a nonmonotonous function of the inverse scattering strength $-1 / V_{\text {imp }}$ and increases in magnitude with 

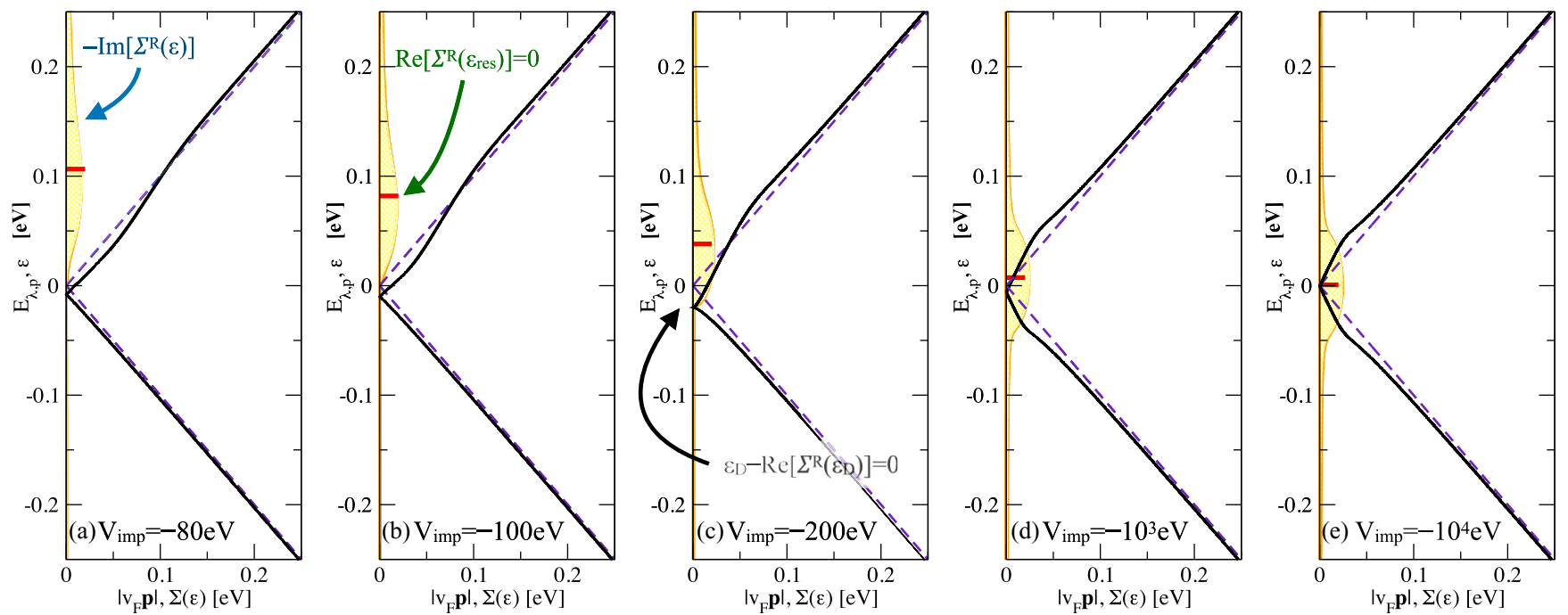

FIG. 1. The panels show how a dilute density of impurities, $n_{\text {imp }}=10^{-4}$, modifies the band structure of graphene. From (a) to (e) the impurity strength is increasing and the values of $V_{\mathrm{imp}}$ are indicated in each panel. The full black line is the impurity-modified band structure, $E_{\lambda, p}=\epsilon-\operatorname{Re} \Sigma_{\text {imp }}^{R}(\epsilon)$, for the different impurity strengths as indicated in each panel. The dashed line is the pristine graphene band structure. Along each $y$ axis we also plot $-\operatorname{Im} \Sigma_{\text {imp }}^{R}(\epsilon)$ which is the impurity induced, frequency dependent, scattering rate which ultimately will give rise to plasmon losses. $-\operatorname{Im} \Sigma_{\text {imp }}^{R}(\epsilon)$ is centered around an impurity resonance $\epsilon_{\text {res }}$, marked by the thick dash on the $y$ axis. We read off $\epsilon_{\text {res }}$ at $\operatorname{Re} \Sigma_{\text {imp }}^{R}\left(\epsilon_{\text {res }}\right)=0$. The final quantity we note in the figures is the shift of the Dirac cone $\epsilon_{\mathrm{D}}$, which is extracted at $\epsilon_{\mathrm{D}}-\operatorname{Re} \Sigma_{\text {imp }}^{R}\left(\epsilon_{\mathrm{D}}\right)=0$.

increasing impurity density. Finally, we plot $-\operatorname{Im} \Sigma_{\text {imp }}^{R}(\epsilon)$ for different scattering strength in Fig. 1. For strong scatterers $-\operatorname{Im} \Sigma_{\mathrm{imp}}^{R}(\epsilon)$ has close to a Lorentzian lineshape around $\epsilon_{\mathrm{res}}$ while for weaker scatterers $-\operatorname{Im} \Sigma_{\text {imp }}^{R}(\epsilon)$ has a wider spread still with a weak maximum at $\epsilon_{\text {res }}$. As $-\operatorname{Im} \Sigma_{\text {imp }}^{R}(\epsilon)$ gives an
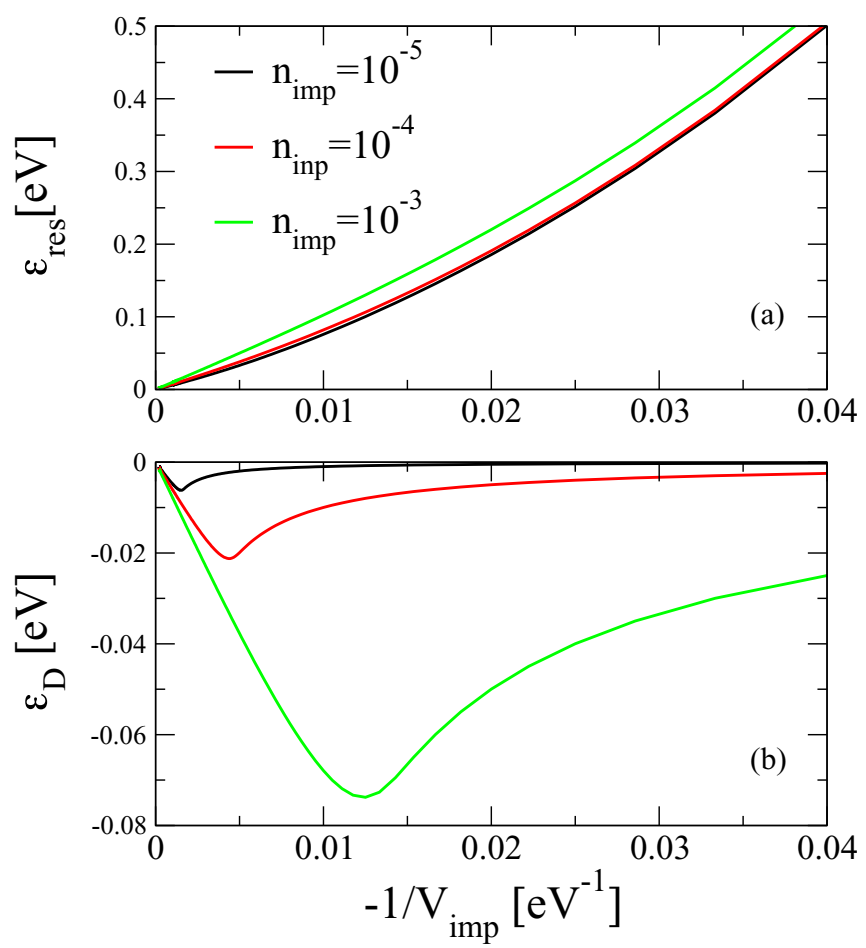

FIG. 2. In panel (a) the location of the impurity resonance is plotted vs $-1 / V_{\text {imp }}$ for three different impurity densities. In panel (b) the shift of the Dirac point $\epsilon_{\mathrm{D}}$ for the same densities. effective frequency-dependent single-particle scattering rate $\Gamma(\epsilon)=\frac{\hbar}{2 \tau(\epsilon)}$, we predict that weak impurities will introduce plasmon losses at all frequencies along the plasmon dispersion while the losses incurred from strong impurities are pronounced when the plasmon mode interferes with $\epsilon_{\text {res }}$.

In this paper, $V_{\text {imp }}$ is chosen to be negative, giving rise to an impurity state in the conduction band, which is a common scenario suggested by first-principle studies [37-42]. All considerations can be repeated for $V_{\text {imp }}>0$ for which the sign of $\epsilon_{\mathrm{res}}$ and $\epsilon_{\mathrm{D}}$ are reversed.

\section{OPTICAL AND TRANSPORT PROPERTIES}

We now focus on the nonlocal graphene conductivity $\sigma(\boldsymbol{q}, \omega)$. With the knowledge of the band structure, and following the method presented in Refs. [35,54], a simplified expression for the conductivity is obtained. The results obtained below are found to be identical if the signs of $E_{F}$ and $V_{\text {imp }}$ are reversed, due to the particle-hole symmetry.

To start out we consider the impact of disorder on the local conductivity $\sigma(\boldsymbol{q}=0, \omega)$. This has been investigated in detail (see, e.g., Refs. [23,24,26,28]). We revisit it nevertheless briefly with attention on how impurities modify the DC conductivity. The impurity contribution to the transport scattering time $\left(\tau_{\mathrm{DC}}\left(E_{F}\right)\right)$ is given by the relation [29]

$$
\frac{1}{\tau_{\mathrm{DC}}\left(E_{F}\right)}=\frac{e^{2} v_{F}^{2}}{2} \frac{\rho\left(E_{F}\right)}{\sigma_{1}(\boldsymbol{q}=0, \omega \mapsto 0)}
$$

in the limit $T \rightarrow 0$. The competition between the density of states and the DC conductivity gives rise to a nontrivial relation between Fermi energy and transport scattering time as shown in Fig. 3. The relaxation time is nonsymmetric in $E_{F}$ for small $V_{\text {imp }}$ and a symmetric behavior is recovered for large $V_{\text {imp }}$. Figure 3 suggests that a chemical potential with the same sign 


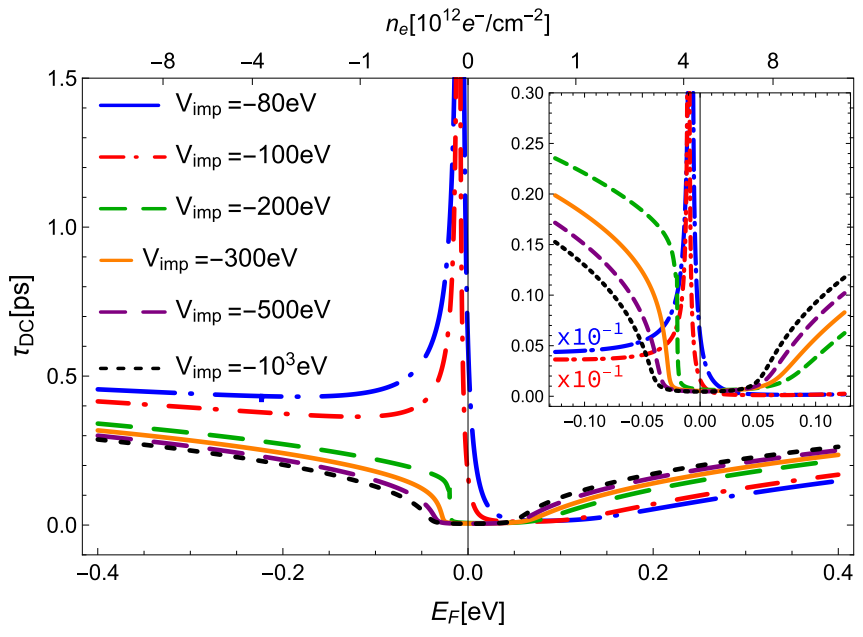

FIG. 3. The relaxation time induced by impurities obtained from equation (10) as a function of the Fermi energy. In the plot, the density of impurities is $n_{\mathrm{imp}}=10^{-4}$; the relaxation time roughly scales as $1 / n_{\text {imp }}$. In the inset is a zoom for small value of $\left|E_{F}\right|$. Different colors and marks correspond to different values of $V_{\text {imp }}$ as indicated by the legend.

as the more common impurity strength may increase $\tau_{\mathrm{DC}}\left(E_{F}\right)$ [55]. For $\left|E_{F}\right|>0.1 \mathrm{eV}$ temperature effects on $\tau_{\mathrm{DC}}\left(E_{F}\right)$ are on the order of $1 \%$ or smaller up to $150 \mathrm{~K}$. The scattering time scales with the density of impurities as $\tau_{\mathrm{DC}}^{-1}\left(E_{F}\right) \propto n_{\text {imp }}$ as expected. In the following we focus mostly on the impurity density order of $n_{\text {imp }} \propto 10^{-4}$. This density of impurities gives a relaxation time that is suitable for plasmonic application in the $\mathrm{THz}$ regime.

Now we turn to the consequence of the impurity band on the nonlocal conductivity. According to the Fermi golden rule, the lossy part of the conductivity $\left(\sigma_{1}\right)$ gives the possibility of the electromagnetic field to release energy to the carriers in graphene by exciting electron-hole pairs [47]. In pristine graphene, there is a triangle in the $(\omega, \boldsymbol{q})$ plane given by $\hbar v_{F} q<\hbar \omega<2 E_{F}-\hbar v_{F} q$, where absorption is forbidden, i.e., $\sigma_{1}(\boldsymbol{q}, \omega)=0$ at $T=0$. Absorption is allowed outside this Pauli-blocked triangle, i.e., for $\hbar \omega \leqslant \hbar v_{F} k$ and $2 E_{F}-$ $\hbar v_{F} k \leqslant \hbar \omega$. The absorption occurs in intraband and interband transitions, respectively $[20,21,47]$. It has also been shown that for pristine graphene the response depends only on one energy scale, the Fermi energy, which scales all energies $\left(\omega, E_{\lambda, p}\right.$ and $\left.k_{B} T\right)$ [47].

In Fig. 4 we plot $\sigma_{1}(\boldsymbol{q}, \omega)$ at $q / k_{F}=0.15$ as a function of frequency $\omega$. The impurity specific features we find are interband processes corresponding to transitions between the impurity band around $\epsilon_{\text {res }}$ and the states around the Fermi energy. The transitions generate an extra peak in $\sigma_{1}(\boldsymbol{q}, \omega)$ at frequencies $\hbar \omega \simeq\left|\epsilon_{\text {res }}-E_{F}\right|$ added to the main peak at $\hbar \omega / E_{F}=0.15$ as seen in Fig. 4. These transitions are single particles excitations [56]. In the lower panel of Fig. 4 an extended range of density of impurities is considered. The extra peak in $\sigma_{1}(\boldsymbol{q}, \omega)$ follows $\epsilon_{\text {res }}$ and is actually a bandlike area in the $(\omega, \boldsymbol{q})$ plane around $\omega \simeq\left|E_{F}-\epsilon_{\text {res }}\right|$ where there are increased losses, independent of $q$. The width of this stripe is given mainly by $-\operatorname{Im} \Sigma_{\text {imp }}^{R}(\epsilon)$. Temperature effects are also important but only at high temperatures. The lower

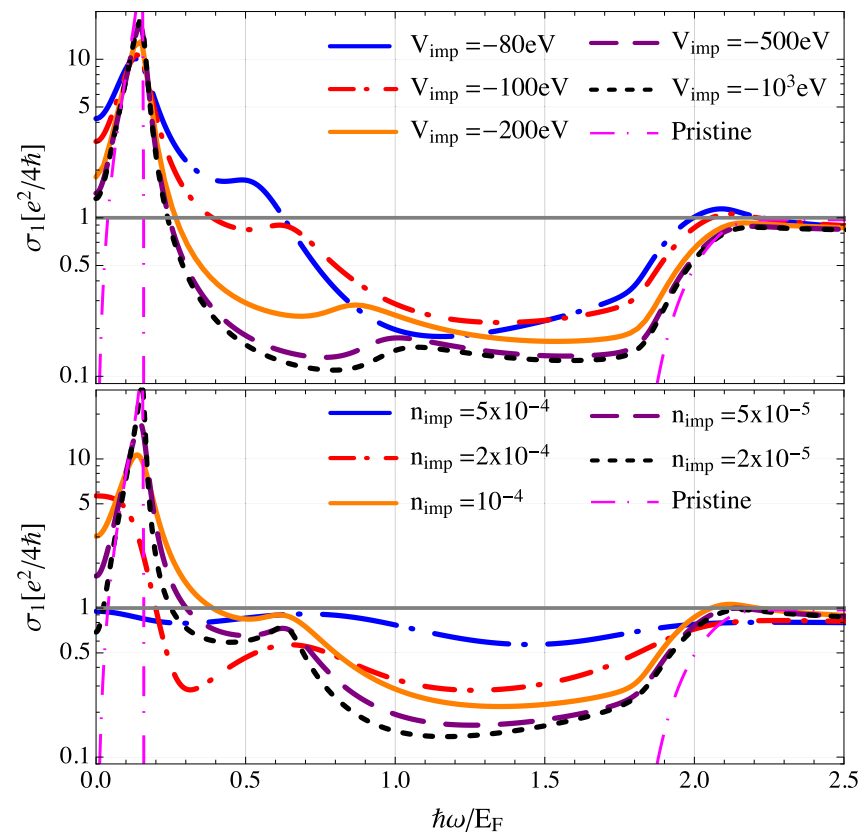

FIG. 4. Real part of the nonlocal conductivity $\sigma_{1}(\boldsymbol{q}, \omega)$ as a function of the frequency. In the top panel the impurity density is set to $n_{\text {imp }}=10^{-4}$ and different lines correspond to different values

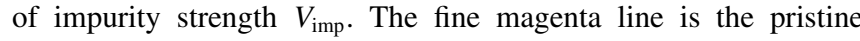
graphene case. Impurities induce peaks in the losses at frequency $0.4 E_{F} \lesssim \hbar \omega \lesssim E_{F}$ for the values of $V_{\text {imp }}$ considered in the figure. The position of the peak depends on $V_{\text {imp }}$, and increasing the strength induces a blueshift of the feature. The lower plot shows the influence of the density of impurity for $V_{\mathrm{imp}}=-100 \mathrm{eV}$. Here, $\sigma_{1}(\boldsymbol{q}, \omega)$ is computed for $T=30 \mathrm{~K}, E_{F}=0.2 \mathrm{eV}$, and $|q|=2 \pi / \lambda$ with $\lambda=$ $130 \mathrm{~nm}\left(q / k_{F} \simeq 0.15\right)$ [7].

panel of Fig. 4 shows how the density of impurities, and hence $-\operatorname{Im} \Sigma_{\text {imp }}^{R}(\epsilon)$, affects transport properties. Since $\Sigma_{\text {imp }}^{R} \propto n_{\text {imp }}$, increasing the impurity density all features in the conductivity are broadened and $\sigma(\boldsymbol{q}, \omega)$ tends to $\sigma_{0}=\mathrm{e}^{2} / 4 \hbar$. For a given $n_{\text {imp }}$, weaker impurities have a stronger effect on AC transport. For the range of parameters explored, the impurity induced peak emerges distinctly above the background at $T<200 \mathrm{~K}$. The new features in $\sigma_{1}$ reflect in a nonmonotonic behavior of $\sigma_{2}$, according to the Kramers-Kronig relations [57]. Similar features are given by the impurity states in the presence of adatoms on the graphene surface [35]. The work presented here confirms that the main features survive in a self-consistent treatment of impurities.

There is a minor mismatch between the computed position of the peak in $\sigma_{1}$ and the estimate $\left|E_{F}-\epsilon_{\text {res }}\right|$. This is due to the impurity-induced energy renormalization $\operatorname{Re} \Sigma_{\text {imp }}^{R}(\epsilon)$ which modifies the band structure. This means that if the real part of the self energy is omitted there are inaccuracies in the values of conductivity and, finally, of resonances and losses of the circuit that embed the graphene sample. In this study it is found that the error in the resonance frequency does not exceed $5 \%$ for $2 \times 10^{-6}<n_{\text {imp }}<2 \times 10^{-4}$.

Now we turn to how the presence of an impurity band affects the plasmonic properties of graphene. As the impurities influence both the dissipative $\left(\sigma_{1}\right)$ and kinetic $\left(\sigma_{2}\right)$ part of the conductance they also affect both the plasmon dispersion 

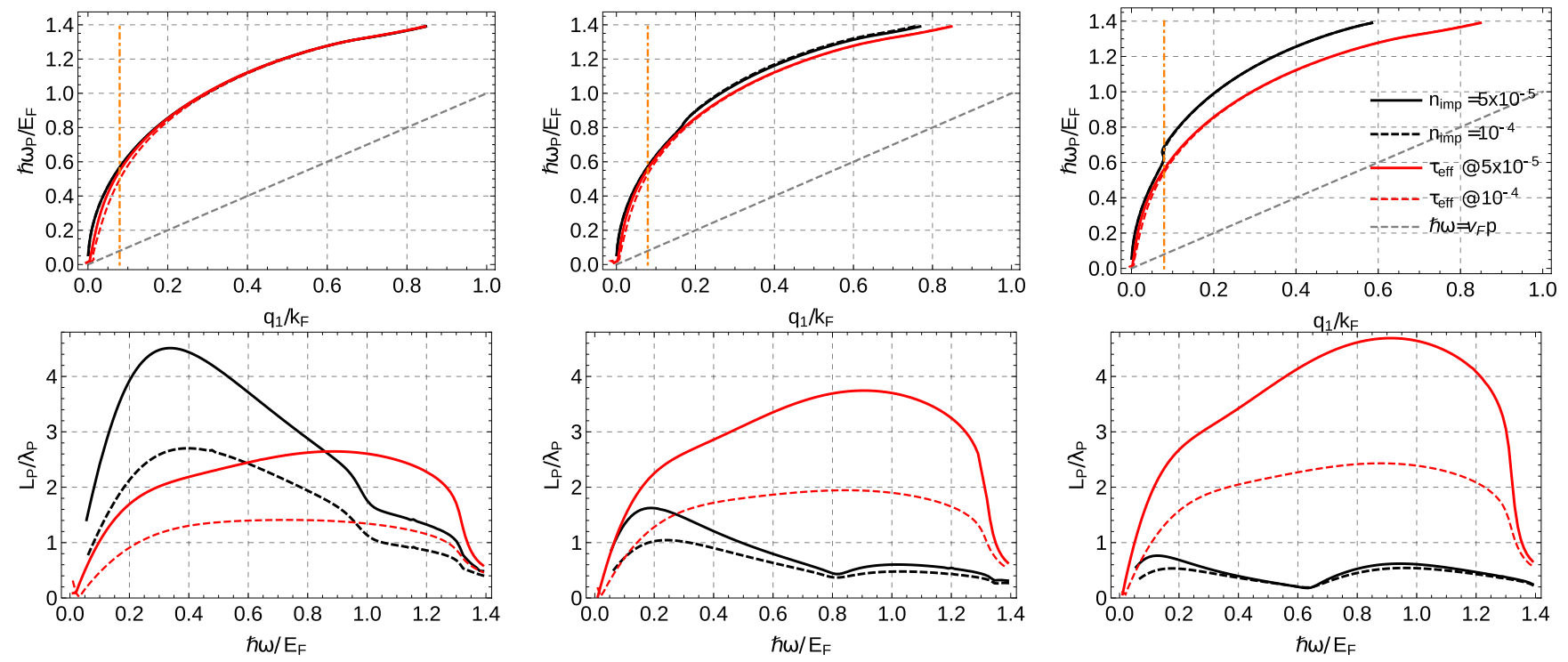

FIG. 5. The plasmon dispersion relation, $\hbar \omega_{P}\left(q_{1}\right)$, and propagation length, $L_{P} / \lambda_{P}=q_{1} /\left(4 \pi q_{2}\right)$ are plotted for different values of impurity strength and density. The impurity strength from the left to the right takes the values $V_{\text {imp }}=-1000,-100,-60 \mathrm{eV}$. The impurity densities $n_{\text {imp }}=10^{-4}$ and $n_{\text {imp }}=5 \times 10^{-5}$ are the dashed and full black lines, respectively. $q_{1,2}$ were computed according to Eq. (2). The plasmon dispersion is given in units of $E_{F} / \hbar$ (here $E_{F}=0.4 \mathrm{eV}$ ). The propagation length is scaled by the plasmon wavelength. The results are compared with relaxation-time approximation results evaluated at the marked scattering times $\tau_{\text {eff }} \cdot \tau_{\text {eff }}$ is chosen so that for each pair $\left(V_{\text {imp }}, n_{\text {imp }}\right)$ the DC relaxation time is extracted from relation (10) and used to compute the dispersion relations according to Ref. [3]. In the top row: The gray dashed line is the single particle continuum $\hbar \omega_{P}=\hbar v_{F} q_{1}$, and the vertical dash-dotted orange line corresponds to the wavelength of $\lambda=130 \mathrm{~nm}$ for $E_{F}=0.4 \mathrm{eV}$. The plots show a strong nontrivial behavior as a function of the parameters $n_{\text {imp }}$ and $V_{\mathrm{imp}}$. The dip in the ratio $L_{P} / \lambda_{p}$ occurs at $\hbar \omega \approx\left|E_{F}-\epsilon_{\text {res }}\right|$ where losses are enhanced.

relation $\omega\left(q_{1}\right)$ and the propagation length $L_{P}=1 /\left(2 q_{2}\right)[58]$. In Fig. 5 the top row shows the plasmon dispersion relation $\omega\left(q_{1}\right)$. The bottom row in Fig. 5 presents the propagation length $L_{P}(\omega)$ in units of the plasmon wavelength $\lambda_{P}=2 \pi / q_{1}$, for the same values of $V_{\text {imp }}$ and $n_{\text {imp }}$ as in the panel directly above. In each column two values of impurity density are shown, $n_{\text {imp }}=$ $5 \times 10^{-5}, 10^{-4}$, and the impurity strength changes with the column. The dispersion relation obtained from the impuritydoped graphene is compared with results from a relaxation time approximation $[3,59]$ using the DC relaxation time value computed according to the finite temperature equivalent of Eq. (10) [29]. The analysis of the losses shows a disagreement between the two approaches as was observed in Refs. $[28,33,60]$ and here confirmed in a self-consistent $\mathbf{t}$-matrix model. The effects of the impurities are fully considered also in the evaluation of the dispersion relation $\omega\left(q_{1}\right)$. As seen in the figure there is quite a discrepancy between the relaxation time approximation and our impurity model. The impurity model shows a clear signature of the impurity resonance as the frequency is swept. This is particularly clear for the strong scattering case. For weaker scatterers we also see a structure in the propagation length at $\epsilon_{\text {res }}$ as well as a shift in the plasmon dispersion at $\hbar \omega=\epsilon_{\text {res }}$. While the relaxation time approximation is able to show damping, it is clear that impurities in the self consistent model contains features that are not captured at all in the relaxation time approximation.

To analyze the dispersion relation in more detail we use a rather large value of the chemical potential $E_{F}=0.4 \mathrm{eV}$ and a temperature of $30 \mathrm{~K}$. The results are shown in Fig. 5 . The purpose of this choice is to enhance the visibility of the effects of the impurity band. Thanks to the approximate scale invariance of the system, the main features remain valid also for smaller values of the Fermi energy. However, the position of the impurity resonance needs to be wisely rescaled and one must keep in mind that the line shape of $-\operatorname{Im} \Sigma_{\text {imp }}^{R}(\epsilon)$ becomes broader the further away $\epsilon_{\text {res }}$ is from the Dirac point. The left panel of Fig. 5 shows the case of strong impurities $\left(V_{\mathrm{imp}}=-10^{3} \mathrm{eV}\right)$; this may represent a graphene lattice with dislocations or holes in it. The impurity resonance is expected around $\epsilon_{\text {res }} \simeq 0.02,0.04 \mathrm{eV}$ for the densities used in the plot $n_{\text {imp }}=5 \times 10^{-5}, 10^{-4}$ (full and dashed lines), respectively. The signature of $\epsilon_{\text {res }}$ is a marked drop in the propagation length, $L_{p} / \lambda_{p}$, at $\hbar \omega / E_{F} \simeq 0.98(0.96)$ for $n_{\text {imp }}=5 \times 10^{-5}\left(10^{-4}\right)$. This brings us to a first conclusion: Holes and dislocations in the graphene crystal reduce the bandwidth of the plasmons to $\hbar \omega<E_{F}$ from the range $\hbar \omega<1.3 E_{F}$ that the relaxation time approximation approach suggests $[3,7]$. We do not consider effects of phonons to underline pure impurity effects. According to the literature an optical phonon introduces an extra bound to the working frequency of graphene plasmons to $\hbar \omega<0.2 \mathrm{eV}$ $[3,61]$. In Fig. 5, the second and third column present the dispersion relation for impurities bands that lie away from the Dirac point. The column in the middle displays the case when $V_{\text {imp }}=-100 \mathrm{eV}$ and corresponds to an impurity band around $\epsilon_{\text {res }} \simeq 0.082 \mathrm{eV}$ and $\epsilon_{\text {res }} \simeq 0.093 \mathrm{eV}$ for $n_{\text {imp }}=5 \times 10^{-5}$ and $n_{\text {imp }}=10^{-4}$, respectively. A clear increase in overall losses appears and close to $\hbar \omega \simeq E_{F}-\epsilon_{\text {res }}$ we see a signature of $\epsilon_{\text {res }}$ as dip in $L_{p}$. In the right column, the dispersion relation for impurities with strength $V_{\mathrm{imp}}=-60 \mathrm{eV}$. Now the impurity band is even higher up in the conductance band compared 

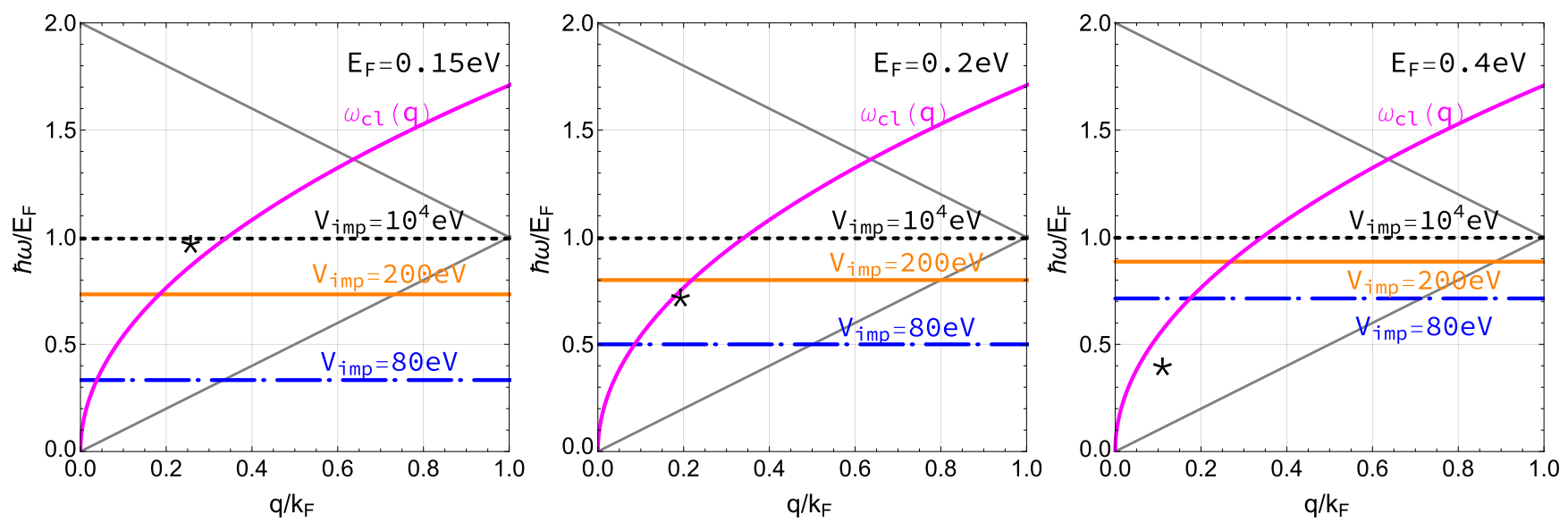

FIG. 6. Plasmon dispersion, in a long wavelength approximation (purple solid line), together with the impurity transitions for different impurity strengths shown for three different chemical potentials. The key insight in the sensing scheme described in Sec. IV is that a fixed light frequency and grating may effectively probe different regions of the plasmon dispersion by use of the gate tunability. Furthermore, this allows the effect of different impurities to be probed since the effect of impurities is largest when the impurity transitions are resonant with the graphene plasmon. The $*$ in the figures mark the point in parameter space that is probed with a light frequency of $0.15 \mathrm{eV}$ and a periodicity of $130 \mathrm{~nm}$ (same as in Sec. IV).

to the case with $V_{\text {imp }}=-100 \mathrm{eV}$ and we find $\epsilon_{\text {res }}=0.15 \mathrm{eV}$ and $\epsilon_{\text {res }}=0.16 \mathrm{eV}$ for the two densities. This reflects the even more lossy conductance and $L_{p} / \lambda_{p} \lesssim 1$ for all frequencies. For large impurity densities, $n_{\text {imp }}>10^{-3}$, the longitudinal plasmons appear to be overdamped according to Eq. (2), and it may not be appropriate to speak about modes. This suggests one obvious reason why graphene of too low quality is not suitable for (longitudinal) plasmonic applications.

The comparison between temperature and finite momenta losses, considered in Ref. [50], and impurity losses reveals that the last are dominating up to room temperature for $\omega / E_{F}<1.2$ and for $n_{\text {imp }}>5 \times 10^{-5}$. At lower density $n_{\text {imp }} \simeq \times 10^{-5}$ the two sources of losses are comparable in the range of frequency $1<\omega / E_{F}<1.4$ and impurity losses are dominant at lower frequency.

\section{PLASMONS AS CHEMICAL SENSING TOOLS}

In the previous section, the effects of impurities on the optical conductivity of graphene and the graphene plasmon resonance were investigated. The effect of the impurities on the plasmons is most pronounced when the plasmon and the impurity transitions are in resonance with each other. This property constitutes an indirect way to transduce optical energy into the impurity band around $\epsilon_{\text {res }}\left(V_{\text {imp }}, n_{\text {imp }}\right)$, in a way that can be specific for a given species of molecules on the surface. One of the main advantages of graphene plasmons is given by the tunability of the optical properties in graphene. By adjusting the Fermi energy in graphene, the plasmon resonance can be tuned [2] in and out of resonance with the impurity transition so that probing the impurity level position becomes possible. This flexibility could be relevant to overcome the constraints introduced by the structure that allows us to couple light and plasmons [47]. Below, a measurement protocol to reconstruct the impurity resonance position is proposed.

The impedance matching required to couple graphene plasmons with light can be achieved by coupling via STM tips $[4,49]$ or by introducing a periodic structure, either a dielectric grating [62] or a patterned graphene sheet [47]. The periodicity fixes the value of the wave vector $\boldsymbol{q}$ to couple the electric field to the plasmons but also reduces the phase space that can be explored, hence the information that can be collected. There are still two degrees of freedom which can be used: the Fermi energy, accessible by gating the graphene, and varying the incident light frequency. In this paper we explore the first possibility, while the second has been discussed in Ref. [35]. The structure of the current-current loss function [47]

$$
S_{j_{\mathrm{x}}}(q, \omega)=\frac{\omega \sigma_{1}(q, \omega)}{\left|1+\frac{i q e^{2} \sigma(q, \omega)}{\omega \varepsilon_{0}\left(\varepsilon_{1}+\varepsilon_{2}\right)}\right|^{2}}
$$

indicates where one may deposit energy in the sample, for instance via electromagnetic radiation. The strongest response is found at sharp maxima in $S_{j_{\mathrm{x}}}(q, \omega)$, and these peaks coincide with the plasmon dispersion. This property has been used in Ref. [63] to map out the dispersion relation of plasmons in graphene. In this paper, we take advantage of the new structures arising in the loss function due to impurity scattering. We use these features to determine the parameter $V_{\text {imp }}$, the value of which represents a certain type of impurity on the graphene surface, and $n_{\text {imp }}$ which is the density of impurities.

Before going to the full numerical results, it is useful to consider a simple model for plasmons and the impurities in order to gain insight into the sensing properties. Figure 6 shows the plasmon dispersion, using the long-wavelength approximation, together with the impurity transitions. This is shown for three different Fermi energies and illustrates how the impurity transitions shift with respect to the plasmon dispersion. The idea is to use this property to distinguish between different positions of the impurity level by observing the graphene plasmon. Indeed, it was shown in Fig. 5 that impurities can severely affect the graphene plasmons by inducing large damping. The left panel in Fig. 7 shows the plasmon dispersion together with the line (black solid line) in parameter space that is probed when varying the Fermi energy 

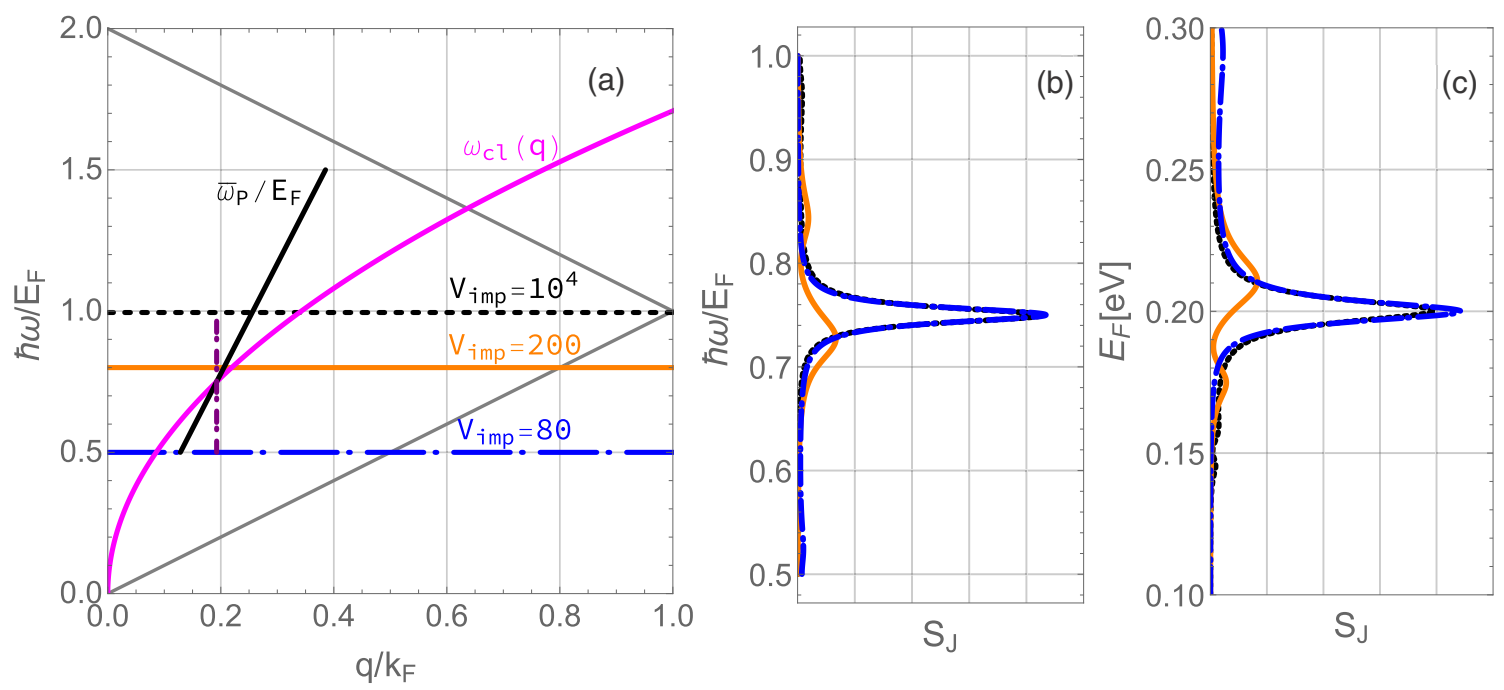

FIG. 7. A simple model is used to gain insight in the interplay between plasmons and impurity levels. The plasmons are here considered in a long wavelength approximation giving rise to a $\sqrt{q}$ behavior of the dispersion (purple solid line). The damping is modeled using a Drude conductivity with an energy dependent scattering time $\tau^{-1}=\tau_{0}^{-1}+\tau(E)^{-1}$ where $\tau(E)^{-1}$ has a Lorentzian shape around the impurity level. (a) Plasmon dispersion relation and impurity transitions shown for $E_{F}=0.2$ (same as middle panel in Fig. 6). The black solid line shows the region probed when changing the Fermi energy from $0.1 \mathrm{eV}$ to $0.3 \mathrm{eV}$ for incident light frequency $0.15 \mathrm{eV}$ and periodicity $130 \mathrm{~nm}$. To highlight the difference between working with a fixed Fermi energy and tunable Fermi energy, the vertical dashed purple line shows a cut in frequency (and $E_{F}=0.2 \mathrm{eV}$ is fixed) which can be obtained by tuning the incidence frequency. (b) The loss function obtained by plotting the loss function following the vertical cut in panel (a). The different colors correspond to having the impurity level at the locations indicated by the horizontal lines in panel (a). (c) The loss function obtained for a fixed light frequency and a fixed grating when changing the Fermi energy, i.e., along the black solid line in panel (a).

from $0.1 \mathrm{eV}$ to $0.3 \mathrm{eV}$. More specifically, the black solid line is obtained by calculating the point in parameter space that is probed for the specific fixed frequency $(0.15 \mathrm{eV})$ and periodic structure (periodicity $130 \mathrm{~nm}$ ) considered. This point moves in the left panel since what is plotted is energy and wave number divided with $E_{F}$ and $k_{F}$ and these quantities are tuned. The vertical purple dashed line shows for comparison a vertical cut made by changing the incident light frequency. These two cuts represent two different ways of probing plasmons in a fixed periodic environment. The loss function obtained for the two cuts are shown in the middle and right panels of the figure. In both panels, the plasmon peak is severely affected by the impurity transition that is resonant with the plasmon at the point in parameter space where it is being probed.

Having obtained some insight from the simple model above, we now consider the full numerical model, and the sensing protocol is suggested as follows. First the periodicity in space $\bar{\lambda}=2 \pi / \bar{q}$ of the electric field is fixed by the periodic structure. This is needed to couple plasmons with the incident light. Quantities with a bar on top, e.g., $\bar{\lambda}$ remain fixed throughout the procedure which is in contrast to the Fermi energy that will be tuned. The light frequency considered is on resonance with the plasmon at a given Fermi energy $\left(\bar{E}_{F}\right)$ for a given reference sample, for example, with dislocations, i.e., $V_{\text {imp }} \mapsto \infty$. The incident light frequency is fixed for the rest of the procedure and denoted with $\bar{\omega}$. The behavior of the loss function while tuning the Fermi energy is recorded to be used as the reference for the next set of measurements. These measurements on further samples with unknown impurity types are done again by recording the loss function values for the same light frequency while varying the Fermi energy as before.
Figure 8 shows the loss function for the full numerical results for various impurity strengths at room temperature. The graphene sample considered in Fig. 8 is assumed to be in vacuum, i.e., suspended. Results for graphene on a substrate are qualitatively similar, however, the plasmon dispersion is somewhat shifted due to the presence of the substrate. The wavelength of the electric field periodicity chosen here is

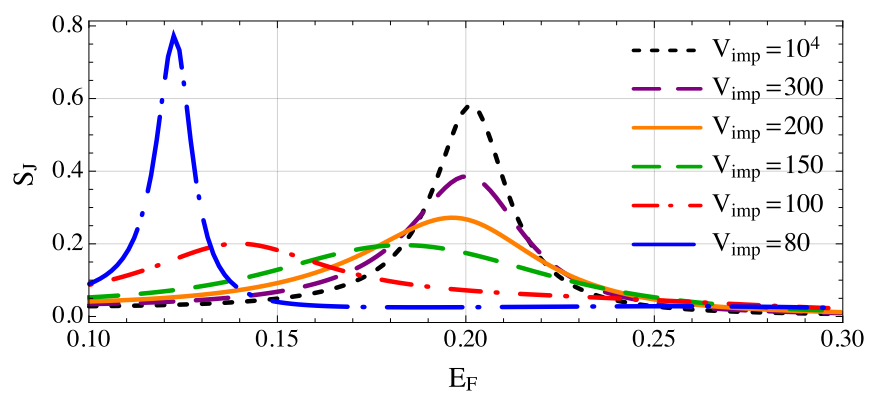

FIG. 8. Loss function plotted for different impurity strengths as a function of the Fermi energy. The impurity density $n_{\mathrm{imp}}$ is $5 \times 10^{-5}$ for all curves. The dashed black line corresponds to the calibrating measurement on a known sample with only dislocations $V_{\text {imp }} \mapsto \infty$. For the other lines the impurity level is moved higher in the conduction band, which can be attributed to unique scattering properties of previously tested chemical compounds. The impurity bands are located at $\epsilon_{\text {res }}=0.046 \mathrm{eV}$ for $V_{\text {imp }}=200 \mathrm{eV}, \epsilon_{\text {res }}=0.056 \mathrm{eV}$ for $V_{\text {imp }}=$ $150 \mathrm{eV}, \epsilon_{\text {res }}=0.082 \mathrm{eV}$ for $V_{\text {imp }}=100 \mathrm{eV}$, and $\epsilon_{\text {res }}=0.1 \mathrm{eV}$ for $V_{\text {imp }}=80 \mathrm{eV}$. In this plot the loss function is evaluated at room temperature and an additional relaxation time of $0.3 \mathrm{ps}$ is introduced to take into account additional relaxation processes. 
equal to $\bar{\lambda}=130 \mathrm{~nm}$, and this corresponds to a plasmon frequency of $\omega_{P} \simeq 0.15 \mathrm{eV}$ for $E_{F}=0.2 \mathrm{eV}$ of the reference sample with dilute densities of impurities $n_{\text {imp }}=5 \times 10^{-5}$ and scattering strength of $V_{\text {imp }}=10^{4} \mathrm{eV}$. In Fig. 8, the plasmonpeak shapes and positions are strongly influenced by the impurity transitions, i.e., the impurity strength $V_{\text {imp }}$. We can from the calculated loss function extract the corresponding impurity properties, which may be a fingerprint of a given chemical compound, assuming known impurity density. While the strength of the impurity scattering shifts the peak position, the density mostly controls the width of the peak. Further analysis is needed to improve the protocol in order to extract both quantities. In this paper we have focused on demonstrating the possibility of sensing microscopic degrees of freedom as well as using the unique possibility given by the tunability of graphene plasmons.

\section{CONCLUSION}

In this paper, a microscopic model of plasmons that considers together impurities [33] and nonlocal effects [15,50] has been developed and analyzed. The present work also contributes to shaping a full microscopic picture of the plasmon in graphene $[34,47]$ with the long term aim to develop further the design of plasmonic devices.

The first-principles computations [37-42] suggest that impurities on the graphene surface introduce an almost flat impurity band close to the Dirac point, whose width and position in energy depend on the type and density of impurities. An impurity model based on the $\mathbf{t}$-matrix formalism in the Green's function framework [23-27] is developed and analyzed. The band structure of the model shows an impurity band similar to first-principles results, Sec. II. The model has two parameters, density and impurity strength, which control the band structure of graphene with impurities. An approximate mapping between the DFT band structure and the theoretical impurity model has been demonstrated to be possible.

It is found here that induced impurity losses have large effects on the optical properties: For frequencies in resonance with the transition from the impurity band to above the chemical potential, a substantial increase of the losses is obtained, Sec. III. This has relevant effects on the dispersion relation for the plasmons. Both the relation $\omega(q)$ and the plasmon damping rate depend on the impurity type. The impurity effect emerges also in the optical conductivity $\sigma(\boldsymbol{q}, \omega)$, and an enhancement of the losses, $\sigma_{1}(\boldsymbol{q}, \omega)$, is observed when the incident light frequency is in resonance with the possible transitions involving the impurity states.

The possibility to identify the type of surface impurities from their effect on the optical response is an avenue that was explored, Sec. IV. In this paper we have shown that the loss function as a function of the Fermi energy has an interesting dependence on the impurity type. Indeed the behavior of the optical conductivity indicates that it may be possible to extract the value of the impurity strength $V_{\text {imp }}$ and the impurity density $n_{\text {imp }}$ and so extract the impurity resonance $\epsilon_{\text {res }}$. Finally the energy of the impurity position can be compared with the result from DFT simulations to determine the chemical compound present on the sample. The proposal for a sensor can take in account realistic laboratory constraints, so that it works at fixed incident light frequency and grating periodicity. This is accomplished using the tunability offered by graphene as a plasmonic material.

\section{ACKNOWLEDGMENTS}

The authors wish to thank the Knut and Alice Wallenberg (KAW) foundation and the Swedish Foundation for Strategic Research (SSF) for financial support. We also thank T. Löfwander for stimulating discussions.
[1] A. Woessner, M. B. Lundeberg, Y. Gao, A. Principi, P. AlonsoGonzález, M. Carrega, K. Watanabe, T. Taniguchi, G. Vignale, M. Polini, J. Hone, R. Hillenbrand, and F. H. L. Koppens, Nat. Mater. 14, 421 (2015).

[2] Z. Q. Li, E. A. Henriksen, Z. Jiang, Z. Hao, M. C. Martin, P. Kim, H. L. Stormer, and D. N. Basov, Nat. Phys. 4, 532 (2008).

[3] M. Jablan, H. Buljan, and M. Soljačić, Phys. Rev. B 80, 245435 (2009).

[4] J. Chen, M. Badioli, P. Alonso-González, S. Thongrattanasiri, F. Huth, J. Osmond, M. Spasenović, A. Centeno, A. Pesquera, P. Godignon, A. Z. Elorza, N. Camara, F. J. F. G. de Abajo, R. Hillenbrand, and F. H. L. Koppens, Nature (London) 487, 77 (2012).

[5] R. Pearce, J. Eriksson, T. Iakimov, L. Hultman, A. L. Spetz, and R. Yakimova, ACS Nano 7, 4647 (2013).

[6] H. Hu, X. Yang, F. Zhai, D. Hu, R. Liu, K. Liu, Z. Sun, and Q. Dai, Nat. Commun. 7, 12334 (2016).

[7] T. Wenger, G. Viola, J. Kinaret, M. Fogelström, and P. Tassin, 2D Mater. 4, 025103 (2017).
[8] A. C. Ferrari, F. Bonaccorso, V. Fal'ko, K. S. Novoselov, S. Roche, P. Bøggild, S. Borini, F. H. L. Koppens, V. Palermo, N. Pugno, J. A. Garrido, R. Sordan, A. Bianco, L. Ballerini, M. Prato, E. Lidorikis, J. Kivioja, C. Marinelli, T. Ryhänen, A. Morpurgo et al., Nanoscale 7, 4598 (2015).

[9] C. W. Chen, S. C. Hung, M. D. Yang, C. W. Yeh, C. H. Wu, G. C. Chi, F. Ren, and S. J. Pearton, Appl. Phys. Lett. 99, 243502 (2011).

[10] F. Bonaccorso, Z. Sun, T. Hasan, and A. C. Ferrari, Nat. Photonics 4, 611 (2010).

[11] F. Schwierz, Nat. Nanotechnol. 5, 487 (2010).

[12] O. Habibpour, Z. S. He, W. Strupinski, N. Rorsman, T. Ciuk, P. Ciepielewski, and H. Zirath, IEEE Microwave and Wireless Components Letters 27, 168 (2017).

[13] S. Thongrattanasiri, A. Manjavacas, and F. J. García de Abajo, ACS Nano 6, 1766 (2012).

[14] T. Christensen, W. Wang, A.-P. Jauho, M. Wubs, and N. A. Mortensen, Phys. Rev. B 90, 241414 (2014).

[15] M. B. Lundeberg, Y. Gao, R. Asgari, C. Tan, B. Van Duppen, M. Autore, P. Alonso-González, A. Woessner, K. Watanabe, 
T. Taniguchi, R. Hillenbrand, J. Hone, M. Polini, and F. H. L. Koppens, Science 357, 187 (2017).

[16] P. T. Araujo, M. Terrones, and M. S. Dresselhaus, Mater. Today 15, 98 (2012).

[17] S. Haldar and B. Sanyal, in Recent Advances in Graphene Research, edited by P. K. Nayak (InTech, Rijeka, 2016), Chap. 10

[18] X. Gong, G. Liu, Y. Li, D. Y. W. Yu, and W. Y. Teoh, Chem. Mater. 28, 8082 (2016).

[19] P. D. Kaushik, I. G. Ivanov, P.-C. Lin, G. Kaur, J. Eriksson, G. Lakshmi, D. Avasthi, V. Gupta, A. Aziz, A. M. Siddiqui, M. Syväjärvi, and G. R. Yazdi, Appl. Surf. Sci. 403, 707 (2017).

[20] B. Wunsch, T. Stauber, F. Sols, and F. Guinea, New J. Phys. 8, 318 (2006)

[21] E. H. Hwang and S. Das Sarma, Phys. Rev. B 75, 205418 (2007).

[22] M. R. Ramezanali, M. M. Vazifeh, R. Asgari, M. Polini, and A. H. MacDonald, J. Phys. A 42, 214015 (2009).

[23] T. Ando, J. Phys. Soc. Jpn. 75, 074716 (2006).

[24] N. M. R. Peres, F. Guinea, and A. H. C. Neto, Phys. Rev. B 73, 125411 (2006).

[25] V. P. Gusynin, S. G. Sharapov, and J. P. Carbotte, Phys. Rev. Lett. 96, 256802 (2006).

[26] N. M. R. Peres, T. Stauber, and A. H. C. Neto, Europhys. Lett. 84, 38002 (2008).

[27] T. Stauber, N. M. R. Peres, and A. H. C. Neto, Phys. Rev. B 78, 085418 (2008).

[28] E. H. Hwang and S. Das Sarma, Phys. Rev. B 77, 195412 (2008).

[29] S. Das Sarma, S. Adam, E. H. Hwang, and E. Rossi, Rev. Mod. Phys. 83, 407 (2011).

[30] M. Jablan, M. Soljačić, and H. Buljan, Phys. Rev. B 89, 085415 (2014).

[31] A. Principi, G. Vignale, M. Carrega, and M. Polini, Phys. Rev. B 88, 195405 (2013).

[32] A. Principi, M. Carrega, M. B. Lundeberg, A. Woessner, F. H. L. Koppens, G. Vignale, and M. Polini, Phys. Rev. B 90, 165408 (2014).

[33] A. Principi, G. Vignale, M. Carrega, and M. Polini, Phys. Rev. B 88, 121405 (2013).

[34] F. Karimi, A. H. Davoody, and I. Knezevic, Phys. Rev. B 93, 205421 (2016).

[35] G. Viola, T. Wenger, J. Kinaret, and M. Fogelström, New J. Phys. 19, 073027 (2017).

[36] D. Novko, Nano Lett. 17, 6991 (2017).

[37] O. Leenaerts, B. Partoens, and F. M. Peeters, Phys. Rev. B 77, 125416 (2008).

[38] T. O. Wehling, K. S. Novoselov, S. V. Morozov, E. E. Vdovin, M. I. Katsnelson, A. K. Geim, and A. I. Lichtenstein, Nano Lett. 8, 173 (2008).

[39] S. Ihnatsenka and G. Kirczenow, Phys. Rev. B 83, 245442 (2011).

[40] M. Gmitra, D. Kochan, and J. Fabian, Phys. Rev. Lett. 110, 246602 (2013).

[41] K. Zollner, T. Frank, S. Irmer, M. Gmitra, D. Kochan, and J. Fabian, Phys. Rev. B 93, 045423 (2016).
[42] T. Frank, S. Irmer, M. Gmitra, D. Kochan, and J. Fabian, Phys. Rev. B 95, 035402 (2017).

[43] E. Economou, Green's Functions in Quantum Physics, Springer Series in Solid-State Sciences (Springer-Verlag, Berlin, Heidelberg, 2006).

[44] T. Löfwander and M. Fogelström, Phys. Rev. B 76, 193401 (2007).

[45] F. Rana, IEEE Transactions on Nanotechnology 7, 91 (2008).

[46] J. D. Buron, D. H. Petersen, P. Boggild, D. G. Cooke, M. Hilke, J. Sun, E. Whiteway, P. F. Nielsen, O. Hansen, A. Yurgens, and P. U. Jepsen, Nano Lett. 12, 5074 (2012).

[47] P. Goncalves and N. Peres, An Introduction to Graphene Plasmonics (World Scientific, Singapore, 2016).

[48] T. R. Zhan, F. Y. Zhao, X. H. Hu, X. H. Liu, and J. Zi, Phys. Rev. B 86, 165416 (2012).

[49] Z. Fei, A. S. Rodin, G. O. Andreev, W. Bao, A. S. McLeod, M. Wagner, L. M. Zhang, Z. Zhao, M. Thiemens, G. Dominguez, M. M. Fogler, A. H. C. Neto, C. N. Lau, F. Keilmann, and D. N. Basov, Nature (London) 487, 82 (2012).

[50] T. Wenger, G. Viola, M. Fogelström, P. Tassin, and J. Kinaret, Phys. Rev. B 94, 205419 (2016).

[51] J. Rammer, Quantum Field Theory of Non-Equilibrium States (Cambridge University Press, Cambridge, 2007).

[52] I. Kupčić, Phys. Rev. B 91, 205428 (2015).

[53] T. O. Wehling, A. M. Black-Schaffer, and A. V. Balatsky, Adv. Phys. 63, 1 (2014).

[54] F. Marsiglio, M. Schossmann, and J. P. Carbotte, Phys. Rev. B 37, 4965 (1988).

[55] According to Ref. [33], impurity effects should be a dominant relaxation process in graphene samples. This supports the statement in the main text that having $\operatorname{sign}\left(E_{F}\right)=\operatorname{sign}\left(V_{\mathrm{imp}}\right)$ can improve DC transport properties. To finally confirm this statement, the effect of other sources of relaxation (e.g., phonon and electron-electron interactions) should also be included at a self-consistent level [34].

[56] For $\epsilon_{\text {res }}-E_{F}<0$, the transitions between single particle states in the impurity band and states above the Fermi energy contribute to an enhancement of losses at this frequency (while for $\epsilon_{\text {res }}-$ $E_{F}>0$ the relevant transitions are from the Fermi surface to the impurity level).

[57] G. F. Giuliani and G. Vignale, Quantum Theory of the Electron Liquid (Cambridge University Press, Cambridge, 2005).

[58] In this paper the propagation length is defined according to Ref. [47] as the distance that the plasmon propagates until the intensity is reduced by a factor $1 / e \simeq 0.37$.

[59] N. D. Mermin, Phys. Rev. B 1, 2362 (1970).

[60] P. Tassin, T. Koschny, and C. M. Soukoulis, Science 341, 620 (2013).

[61] K. Ishikawa and T. Ando, J. Phys. Soc. Jpn. 75, 084713 (2006).

[62] X. Zhu, W. Yan, P. U. Jepsen, O. Hansen, N. A. Mortensen, and S. Xiao, Appl. Phys. Lett. 102, 131101 (2013).

[63] H. Yan, T. Low, W. Zhu, Y. Wu, M. Freitag, X. Li, F. Guinea, P. Avouris, and F. Xia, Nat. Photonics 7, 394 (2013). 